TAIWANESE JOURNAL OF MATHEMATICS

Vol. 16, No. 2, pp. 531-543, April 2012

This paper is available online at http://journal.taiwanmathsoc.org.tw

\title{
FROM STEINER TRIPLE SYSTEMS TO 3-SUN SYSTEMS
}

\author{
Chin-Mei Fu, Nan-Hua Jhuang, Yuan-Lung Lin and Hsiao-Ming Sung
}

\begin{abstract}
An $n$-sun is the graph with $2 n$ vertices consisting of an $n$-cycle with $n$ pendent edges which form a 1 -factor. In this paper we show that the necessary and sufficient conditions for the decomposition of complete tripartite graphs with at least two partite sets having the same size into 3 -suns and give another construction to get a 3 -sun system of order $n$, for $n \equiv 0,1,4,9(\bmod$ 12). In the construction we metamorphose a Steiner triple system into a 3 -sun system. We then embed a cyclic Steiner triple system of order $n$ into a 3 -sun system of order $2 n-1$, for $n \equiv 1(\bmod 6)$.
\end{abstract}

\section{INTRODUCTION}

A decomposition of a graph $G$ is a set $\mathbb{H}=\left\{H_{1}, H_{2}, \ldots, H_{t}\right\}$ of subgraphs of $G$ such that $E\left(H_{1}\right) \cup E\left(H_{2}\right) \cup \cdots \cup E\left(H_{t}\right)=E(G)$ and $E\left(H_{i}\right) \cap E\left(H_{j}\right)=\emptyset$ for $i \neq j$. For convenience, we say that $G$ can be decomposed into $H_{1}, H_{2}, \cdots, H_{t}$. If $H_{i}$ is isomorphic to a graph $H$ for each $i=1,2, \ldots, t$, then we say that $G$ has an $H$ decomposition. A Steiner triple system of order $n$ (more simply triple system) is a pair $(X, T)$ where $X$ is an $n$-set and $T$ is a collection of edge disjoint triangles (or triples) which partition the edge set of $K_{n}$ with the vertex set $X$. It is well known [3] that the spectrum for Steiner triple systems(STS) is precisely the set of all $n \equiv 1$ or 3 (mod 6). A 3-sun is a graph with six vertices $a, b, c, d, e, f$ consisting of a triangle $(a, b, c)$ and a 1-factor $\{\{a, d\},\{b, e\},\{c, f\}\}$. We will denote this 3 -sun by $(a, b, c ; d, e, f)$. A 3-sun system of order $n,(3 S S(n))$, is a pair $(Y, S)$ where $Y$ is an $n$-set and $S$ is a collection of edge disjoint 3-suns which partition the edge set of $K_{n}$ with the vertex set $Y$. In [6], Yin had shown that the spectrum for 3-sun system is precisely $n \equiv 0,1,4,9(\bmod 12)$ and if $(Y, S)$ is a 3 -sun system of order $n$ then $|S|=n(n-1) / 12$.

Received May 17, 2010, accepted December 18, 2010.

Communicated by Hung-Lin Fu.

2010 Mathematics Subject Classification: 05B30.

Key words and phrases: Steiner triple system, 3-Sun, 3-Sun system, Cyclic, Decomposition. This research is supported by NSC 98-2115-M-032-005-MY3. 
In this paper we give the different constructions to get 3 -sun systems of order $n$. Since a 3 -sun is a tripartite graph, in Section 2, we consider the decomposition of a complete tripartite graph $K_{p, p, r}$ into 3 -suns. We obtain that the necessary and sufficient conditions for the existence of a decomposition of $K_{p, p, r}$ into 3 -suns. In Section 3, we use recursive construction to construct 3 -sun systems of order $n$ when $n \equiv 0,4(\bmod 12)$. For $n \equiv 1(\bmod 12)$, we construct a cyclic 3 -sun system of order $n$. For $n \equiv 9(\bmod 12)$, we metamorphose a Kirkman triple system(KTS) of order $n$ into a 3 -sun system of order $n$. Clearly the triangles of a 3 -sun system cannot form a triple system. So the following problem is immediate. What is the largest cyclic Steiner triple system can be embedded in the partial triple system consisting of the triangles of a 3-sun system? In Section 4, we embed a cyclic Steiner triple system of order $6 m+1$ into a 3 -sun system of order $12 m+1$.

\section{DeCompose $K_{p, q, r}$ INTO 3 -Suns}

Let $p, q, r$ be positive integers. For convenience, we will let $A=\left\{a_{1}, a_{2}, \cdots, a_{p}\right\}$, $B=\left\{b_{1}, b_{2}, \cdots, b_{q}\right\}, C=\left\{c_{1}, c_{2}, \cdots, c_{r}\right\}$ be three partite sets of $K_{p, q, r}$.

Lemma 2.1. Let $p, q, r$ be positive integers and $p \geq q \geq r$. If $K_{p, q, r}$ has $a$ 3-sun decomposition, then $6 \mid(p q+q r+p r)$ and $r \geq \max \left\{\frac{p}{3}, \frac{p q}{p+q}\right\}$.

Proof. If $K_{p, q, r}$ has a 3 -sun decomposition, then $6 \mid(p q+q r+p r)$ and there are $\frac{p q+q r+p r}{6} 3$-suns. Since a 3 -sun has three vertices of degree 3 and each belongs to different partite sets, we have $(p+q) r \geq 3 \cdot \frac{p q+q r+p r}{6}$. It implies that $(p+q) r \geq p q$, thus $r \geq \frac{p q}{p+q}$. Since $K_{p, q, r}$ can be decomposed into at most $q r$ 3 -suns, we have $\frac{p q+q r+p r}{6} \leq q r$. Combining the inequality $(p+q) r \geq p q$, we obtain that $r \geq \frac{p}{3}$. Therefore, $r \geq \max \left\{\frac{p}{3}, \frac{p q}{p+q}\right\}$.

If $p=q=r$, then $K_{p, q, r}$ has a 3 -sun decomposition provided that $p$ is even. For example, $K_{2,2,2}$ can be decomposed into two 3 -suns: $\left(a_{1}, b_{1}, c_{1} ; b_{2}, c_{2}, a_{2}\right)$ and $\left(a_{2}, b_{2}, c_{2} ; b_{1}, c_{1}, a_{1}\right)$. Since $K_{n, n, n}$ can be decomposed into $n^{2}$ triangles from a Latin square of order $n$, we obtain that $K_{p, p, p}$ has a 3 -sun decomposition if and only if $p$ is even.

Next we will consider the decomposition of $K_{p, p, r}$.

Lemma 2.2. Let $p \geq 2$ and $r \geq 2$ be integers. If $K_{p, p, r}$ has a 3 -sun decomposition, then $\frac{p}{2} \leq r \leq \frac{5 p}{2}$ and $(1) p \equiv 0(\bmod 6),(2) p \equiv 2(\bmod 6), r \equiv 2$ $(\bmod 3)$, or $(3) p \equiv 4(\bmod 6), r \equiv 1(\bmod 3)$.

Proof. By counting the number of edges of $K_{p, p, r}$, if $K_{p, p, r}$ has a 3 -sun decomposition, then $6 \mid p(p+2 r)$. It follows that $p$ should be even and $3 \mid p$ or $3 \mid p+2 r$. This implies either $p \equiv 0(\bmod 6), p \equiv 2(\bmod 6)$ and $r \equiv 2(\bmod 3)$ or 
$p \equiv 4(\bmod 6)$ and $r \equiv 1(\bmod 3)$. If $p \geq r$, by Lemma $2.1, r \geq \max \left\{\frac{p}{3}, \frac{p^{2}}{p+p}\right\}$, we have $r \geq \frac{p}{2}$. If $r \geq p$, then $K_{r, p, p}$ can be decomposed into at most $p^{2} 3$-suns. We have $p^{2} \geq \frac{p^{2}+2 p r}{6}$, thus $r \leq \frac{5 p}{2}$. Combining above two results, we obtain $\frac{p}{2} \leq r \leq \frac{5 p}{2}$.

Lemma 2.3. Let $p$ be even. If $K_{p, p, s}$ and $K_{p, p, t}$ have 3 -sun decompositions, then $K_{n p, n p, m s+(n-m) t}$ has a 3-sun decomposition for $0 \leq m \leq n$.

Proof. The first two partite sets of $K_{n p, n p, m s+(n-m) t}$ can be partitioned into $n$ groups each group containing $p$ elements and the third partite set can be partitioned into $n$ groups, $m$ of them containing $s$ elements, the others containing $t$ elements. Since $K_{n, n, n}$ can be decomposed into $n^{2}$ triangles from a Latin square of order $n, K_{n p, n p, m s+(n-m) t}$ can be decomposed into $n^{2}$ triangles and each triangle corresponds to a $K_{p, p, t}$ or a $K_{p, p, s}$. Thus $K_{n p, n p, m s+(n-m) t}$ can be decomposed into $n m$ copies of $K_{p, p, s}$ and $n(n-m)$ copies of $K_{p, p, t}$. Since $K_{p, p, s}$ and $K_{p, p, t}$ have a 3 -sun decomposition respectively, $K_{n p, n p, m s+(n-m) t}$ has a 3 -sun decomposition.

Lemma 2.4. Let $p$ and $r$ be positive integers. If $p \equiv 2(\bmod 6), r \equiv 2$ $(\bmod 3)$, or $p \equiv 4(\bmod 6), r \equiv 1(\bmod 3)$, and $\frac{p}{2} \leq r \leq \frac{5 p}{2}$, then $K_{p, p, r}$ has $a$ 3-sun decomposition.

Proof.

(1) $p \leq r \leq \frac{5 p}{2}$. By Lemma 2.2, if $K_{2,2, r}$ has a 3 -sun decomposition, then $r=2$ or 5. $K_{2,2,2}$ has already been done. We can decompose $K_{2,2,5}$ into four 3 -suns: $\left(a_{1}, b_{1}, c_{1} ; c_{4}, c_{5}, b_{2}\right),\left(a_{1}, b_{2}, c_{2} ; c_{5}, c_{3}, a_{2}\right),\left(a_{2}, b_{1}, c_{3} ; c_{5}, c_{2}, a_{1}\right),\left(a_{2}, b_{2}, c_{4}\right.$; $\left.c_{1}, c_{5}, b_{1}\right)$. By using the decomposition of $K_{2,2,2}$ and $K_{2,2,5}$, we can get the following construction. Let $k$ be a positive integer. If $p=6 k+2=(3 k+1) \cdot 2$ and $r=3 t+2 \leq 15 k+5$, let $m=5 k-t+1$, then $r=m \cdot 2+(3 k+1-m) \cdot 5$. By Lemma 2.3, $K_{6 k+2,6 k+2, r}$ has a 3 -sun decomposition. If $p=6 k+4=(3 k+2) \cdot 2$ and $r=3 t+1 \leq 15 k+10$, let $m=5 k-t+3$, then $r=m \cdot 2+(3 k+2-m) \cdot 5$. By Lemma 2.3, $K_{6 k+4,6 k+4, r}$ has a 3 -sun decomposition.

(2) $\frac{p}{2} \leq r<p$. We can decompose $K_{p, p, r}$ into 3 -suns as follows.

Let $s=\left\lfloor\frac{r}{3}\right\rfloor$. Then $\left\lfloor\frac{p}{6}\right\rfloor \leq s \leq\left\lfloor\frac{p}{3}\right\rfloor$. Let $q=\left\lfloor\frac{p}{3}\right\rfloor-s$.

(i) For $m=1,2, \ldots, q, i=0,1,2, \ldots, p-1$,

$\left(a_{2 m-1+i}, b_{1+i}, c_{m+i} ; b_{3 m-1+i}, a_{2 m+i}, a_{\frac{p}{2}+i}\right)$ and

$\left(a_{2 q+2 m-1+i}, b_{1+i}, c_{q+m+i} ; b_{3 q+3 m-1+i}, a_{2 q+2 m+i}, b_{q+2 m+i}\right)$.

Notice that the indices of $a$ and $b$ are restricted to $Z_{p}=\{1,2, \ldots, p\}$ and the indices of $c$ are restricted to $Z_{\frac{p}{2}}=\left\{1,2, \ldots, \frac{p}{2}\right\}$

(ii) For $m=1,2, \ldots, r-\frac{p}{2}, j=0,1,2, \ldots, \frac{p}{2}-1$, $\left(a_{4 q+2 m-1+j}, b_{1+j}, c_{\frac{p}{2}+m} ; c_{2 q+m+j}, a_{4 q+2 m+j}, b_{\frac{p}{2}+1+j}\right)$ and 
$\left(a_{4 q+2 m-1+j^{\prime}}, b_{1+j^{\prime}}, c_{2 q+m+j^{\prime}} ; c_{\frac{p}{2}+m}, a_{4 q+2 m+j^{\prime}}, b_{\frac{p}{2}+1+j^{\prime}}\right), j^{\prime}=\frac{p}{2}, \frac{p}{2}+$ $1, \ldots, p-1$.

Notice that the indices of $a$ and $b$ are restricted to $Z_{p}$ and the values of $2 q+m+j$ and $2 q+m+j^{\prime}$ are restricted to $Z_{\frac{p}{2}}$.

Lemma 2.5. Let $p$ and $r$ be positive integers. If $p \equiv 0(\bmod 6)$ and $p / 2 \leq$ $r \leq 5 p / 2$, then $K_{p, p, r}$ has a 3-sun decomposition.

Proof.

(1) If $p=6$, then $3 \leq r \leq 15$. Combining $K_{2,2,2}$ and $K_{2,2,5}$, by Lemma 2.3, we can get that $K_{6,6, r}$ has a 3 -sun decomposition for $r=6,9,12,15$, For the rest of $r$, the decomposition of $K_{6,6, r}$ can be found in Appendix.

(2) Let $k \geq 2$ be a positive integer. If $p=6 k$, then $3 k \leq r \leq 15 k$. Let $i=\left\lfloor\frac{r}{k}\right\rfloor$ and $m=r-i k \geq 0$, then $r$ can be written as $m(i+1)+(k-m) i$. By (1) and Lemma 2.3, we obtain that $K_{p, p, r}$ has a 3 -sun decomposition.

By Lemma 2.2, 2.4 and 2.5, we obtain

Theorem 2.6. Let $p$ and $r$ be positive integers. $K_{p, p, r}$ has a 3-sun decomposition if and only if $\frac{p}{2} \leq r \leq \frac{5 p}{2}$ and $(1) p \equiv 0(\bmod 6),(2) p \equiv 2(\bmod 6), r \equiv 2$ $(\bmod 3)$, or $(3) p \equiv 4(\bmod 6), r \equiv 1(\bmod 3)$.

We close this section by decomposing $K_{2 n, 2 n, 2 n}$ into cyclic 3 -suns. Let $A, B$, and $C$ be three partite sets of $K_{2 n, 2 n, 2 n} . K_{2 n, 2 n, 2 n}$ can be decomposed into cyclic 3 -suns if there is an automorphism which is a permutation with three orbits and each orbits has length $2 n$, see [4]. Let $t=\left(a_{i}, b_{j}, c_{k} ; b_{u}, c_{v}, a_{w}\right)$ be a 3 -sun in $K_{2 n, 2 n, 2 n}$, where $a_{i}, a_{w} \in A, b_{j}, b_{u} \in B$, and $c_{k}, c_{v} \in C$. We define $d_{A B}(t)=$ $\left\{0_{j-i}, 0_{u-i}\right\}, d_{B C}(t)=\left\{1_{k-j}, 1_{v-j}\right\}, d_{C A}(t)=\left\{2_{i-k}, 2_{w-k}\right\}$, the indices are taken modulo $2 n$. Let $d(t)=d_{A B}(t) \cup d_{B C}(t) \cup d_{C A}(t)$. We call $d(t)$ is the difference set of $t=\left(a_{i}, b_{j}, c_{k} ; b_{u}, c_{v}, a_{w}\right)$. Let $D(H)=\{d(t) \mid t \in H\}$, where $H$ is a collection of 3 -suns in $K_{2 n, 2 n, 2 n}$. If $T$ contains $n 3$-suns in $K_{2 n, 2 n, 2 n}$ and $D(T)=\left\{0_{i}, 1_{i}, 2_{i} \mid i=0,1, \cdots, 2 n-1\right\}$, then we call that $T$ is a set of base 3 -suns in $K_{2 n, 2 n, 2 n}$. That is, $K_{2 n, 2 n, 2 n}$ can be decomposed into cyclic 3 -suns $\bigcup_{x=0}^{2 n-1}(T+x)=\left\{\left(a_{i+x}, b_{j+x}, c_{k+x} ; b_{u+x}, c_{v+x}, a_{w+x}\right) \mid\left(a_{i}, b_{j}, c_{k} ; b_{u}, c_{v}, a_{w}\right) \in\right.$ $T, x=0,1, \cdots, 2 n-1\}$. The indices of $a, b$, and $c$ are taken modulo $2 n$. For example, if $T=\left\{\left(a_{1}, b_{1}, c_{1} ; b_{2}, c_{2}, a_{2}\right)\right\}$ and $D(T)=\left\{0_{0}, 0_{1}, 1_{0}, 1_{1}, 2_{0}, 2_{1}\right\}$, then $K_{2,2,2}$ can be decomposed into cyclic 3 -suns. Therefore, if we can find the base 3 -suns in $K_{2 n, 2 n, 2 n}$, then $K_{2 n, 2 n, 2 n}$ can be decomposed into cyclic 3 -suns.

Theorem 2.7. Let $n$ be a positive integer. $K_{2 n, 2 n, 2 n}$ can be decomposed into cyclic 3-suns.

Proof. Construct the base 3 -suns in $K_{2 n, 2 n, 2 n}$ as follows:

The following indices of $a, b$, and $c$ are restricted to the set $\{1,2, \ldots, 2 n\}$. 
(1) $n$ is odd. Let $m=\frac{n-1}{2}$,

(i) $k=0,1, \ldots, m,\left(a_{1}, b_{4 k+1}, c_{n+2 k+1} ; b_{4 k+2}, c_{1}, a_{n-2 k+1}\right) \in T$.

(ii) $k=0,1, \ldots, m-1,\left(a_{1}, b_{4 k+3}, c_{2 k+2} ; b_{4 k+4}, c_{1}, a_{2 n-2 k}\right) \in T$.

We have $D(T)=\left\{0_{4 k}, 0_{4 k+1}, 1_{n-2 k}, 1_{2 n-4 k}, 2_{n-2 k}, 2_{2 n-4 k} \mid k=0,1, \ldots, m\right\}$ $\cup\left\{0_{4 k+2}, 0_{4 k+3}, 1_{2 n-2 k-1}, 1_{2 n-4 k-2}, 2_{2 n-2 k-1}, 2_{2 n-4 k-2} \mid k=0,1, \ldots, m-\right.$ $1\}=\left\{0_{i}, 1_{i}, 2_{i} \mid i=0,1, \ldots, 2 n-1\right\}$.

(2) $n$ is even. Let $m=\frac{n}{2}$,

(i) $\left(a_{1}, b_{1}, c_{n+1} ; b_{2}, c_{1}, a_{n+1}\right) \in T$.

(ii) $k=1,2, \ldots, m-1,\left(a_{1}, b_{4 k+1}, c_{n+2 k+1} ; b_{4 k+2}, c_{n+6 k+1}, a_{4 k+1}\right) \in T$.

(iii) $k=0,1, \ldots, m-1,\left(a_{1}, b_{4 k+3}, c_{2 k+2} ; b_{4 k+4}, c_{6 k+4}, a_{4 k+3}\right) \in T$.

We have $D(T)=\left\{0_{0}, 0_{1}, 1_{n}, 1_{0}, 2_{n}, 2_{0}\right\} \cup\left\{0_{4 k}, 0_{4 k+1}, 1_{n-2 k}, 1_{n+2 k}, 2_{n-2 k}\right.$, $\left.2_{n+2 k} \mid k=1,2, \ldots, m-1\right\} \cup\left\{0_{4 k+2}, 0_{4 k+3}, 1_{2 n-2 k-1}, 1_{2 k+1}, 2_{2 n-2 k-1}, 2_{2 k+1}\right.$ $\mid k=0,1,2, \ldots, m-1\}=\left\{0_{i}, 1_{i}, 2_{i} \mid i=0,1, \ldots, 2 n-1\right\}$.

Therefore, $K_{2 n, 2 n, 2 n}$ can be decomposed into cyclic 3 -suns.

\section{3-Sun System OF ORder $n$}

In this section, we will construct the 3 -sun system of order $n$, $3 S S(n)$, i.e., decomposing $K_{n}$ into 3 -suns. The spectrum of $3 S S(n)$ is precisely $n \equiv 0,1,4,9$ $(\bmod 12)$. First we will see the construction of $3 S S(n)$ for $n \equiv 0,4(\bmod 12)$, by using the decomposition of complete tripartite graphs into 3 -suns. Let the vertex set of $K_{n}$ be $\{1,2, \ldots, n\}$.

\section{Example 3.1.}

(a) $3 S S(12)=\{(1,3,4 ; 9,11,12),(1,5,11 ; 2,8,12),(1,7,10 ; 8,12,3)$, $(2,6,12 ; 4,5,10),(2,8,11 ; 5,9,4),(3,5,12 ; 6,10,1),(3,7,9 ; 2,5,12)$, $(4,6,9 ; 7,11,2),(4,8,10 ; 5,12,2),(6,7,8 ; 1,2,3),(9,10,11 ; 5,6,7)\}$.

(b) $3 S S(24)=\{(1,2,4 ; 8,9,23),(1,3,7 ; 16,24,8),(1,5,6 ; 22,8,13)$, $(1,9,21 ; 10,17,5),(1,11,18 ; 12,20,3),(1,13,23 ; 14,19,5),(2,3,5 ; 8,10,18)$, $(2,6,7 ; 21,8,14),(2,10,22 ; 11,18,6),(2,12,19 ; 13,21,4),(2,14,24 ; 15,20,6)$, $(3,4,6 ; 8,11,17),(3,11,23 ; 12,19,7),(3,15,17 ; 16,21,7),(3,13,20 ; 14,22,5)$, $(4,5,7 ; 8,12,20),(4,12,24 ; 13,20,8),(4,14,21 ; 15,23,6),(4,16,18 ; 9,22,8)$, $(5,9,19 ; 10,23,1),(5,13,17 ; 14,21,1),(5,15,22 ; 16,24,7),(6,10,20 ; 11,24,2)$, $(6,14,18 ; 15,22,2),(6,16,23 ; 9,17,8),(7,11,21 ; 12,17,3),(7,15,19 ; 16,23,3)$, $(7,9,24 ; 10,18,1),(8,12,22 ; 13,18,4),(8,16,20 ; 9,24,4),(8,14,19 ; 15,17,6)$, $(8,10,17 ; 11,19,2),(9,10,12 ; 14,21,6),(9,11,16 ; 20,5,14),(9,13,15 ; 3,14,18)$, $(10,11,13 ; 14,22,7),(10,15,16 ; 4,14,19),(11,12,15 ; 14,23,1)$, $(12,13,16 ; 14,24,2),(17,18,21 ; 19,13,8),(17,22,23 ; 4,19,10)$, $(17,20,24 ; 12,1,19),(18,20,22 ; 19,15,3),(18,23,24 ; 7,19,11)$, $(20,21,23 ; 19,16,2),(21,22,24 ; 19,9,5)\}$. 
Lemma 3.2. If $n \equiv 0(\bmod 12)$, then there exists a 3 -sun system of order $n$.

Proof. By Example 3.1, there are 3-sun systems of order 12 and 24 respectively. Let $n=12 m$ where $m \geq 3$. Let $m=3 s+p$ where $s \geq 1$ and $0 \leq p \leq 2 . K_{n}$ can be viewed as the union of two $K_{12 s}$ 's, one $K_{12 s+12 p}$ and one $K_{12 s, 12 s, 12 s+12 p}$. By Lemma 2.5, $K_{12 s, 12 s, 12 t}$ can be decomposed into 3 -suns if $\frac{s}{2} \leq t \leq \frac{5 s}{2}$. By Example 3.1 and the above construction, $K_{n}$ can be recursively decomposed into 3 -suns as $n>24$, except $n=60$. Since $K_{60}$ can be viewed as the union of one $K_{12}$, two $K_{24}$ 's and one $K_{24,24,12}, K_{60}$ has a 3 -sun system. The proof is completed.

\section{Example 3.3.}

(a) $3 S S(16)=\{(1,2,4 ; 13,8,11),(1,5,9 ; 6,12,13)$, $(1,14,15 ; 8,3,5),(1,3,16 ; 7,10,5),(2,3,5 ; 14,9,13),(2,6,10 ; 7,13,14)$, $(2,15,16 ; 9,4,6),(3,4,6 ; 15,10,14),(3,7,11 ; 8,14,15),(4,5,7 ; 16,11,15)$, $(4,8,12 ; 9,15,16),(5,6,8 ; 10,12,16),(6,7,9 ; 11,13,16),(7,8,10 ; 12,14,1)$, $(8,9,11 ; 13,15,2),(9,10,12 ; 14,16,3),(10,11,13 ; 15,1,4),(11,12,14 ; 16,2,5)$, $(12,13,15 ; 1,3,6),(13,14,16 ; 2,4,7)\}$.

(b) $3 S S(28)=\{(1,2,4 ; 22,10,14),(1,3,28 ; 9,13,6),(1,6,25 ; 13,19,8)$, $(1,5,10 ; 12,17,23),(1,8,15 ; 7,19,22),(1,20,24 ; 14,3,8),(1,26,27 ; 11,4,7)$, $(2,3,5 ; 23,11,15),(2,6,11 ; 13,18,24),(2,7,26 ; 14,20,9),(2,9,16 ; 8,20,23)$, $(2,21,25 ; 15,4,9),(2,27,28 ; 12,5,8),(3,4,6 ; 24,12,16),(3,7,12 ; 14,19,25)$, $(3,8,27 ; 15,21,10),(3,10,17 ; 9,21,24),(3,22,26 ; 16,5,10),(4,5,7 ; 25,13,17)$, $(4,8,13 ; 15,20,26),(4,11,18 ; 10,22,25),(4,23,27 ; 17,6,11),(4,9,28 ; 16,22,11)$, $(5,6,8 ; 26,14,18),(5,9,14 ; 16,21,27),(5,12,19 ; 11,23,26),(5,24,28 ; 18,7,12)$, $(6,7,9 ; 27,15,19),(6,10,15 ; 17,22,28),(6,13,20 ; 12,24,27),(7,8,10 ; 28,16,20)$, $(7,11,16 ; 18,23,1),(7,14,21 ; 13,25,28),(8,9,11 ; 14,17,21),(8,12,17 ; 22,24,2)$, $(9,10,12 ; 15,18,22),(9,13,18 ; 23,25,3),(10,11,13 ; 16,19,23)$, $(10,14,19 ; 24,26,4),(11,12,14 ; 17,20,24),(11,15,20 ; 25,27,5)$, $(12,13,15 ; 18,21,25),(12,16,21 ; 26,28,6),(13,14,16 ; 19,22,26)$, $(13,17,22 ; 27,1,7),(14,15,17 ; 20,23,27),(14,18,23 ; 28,2,8)$, $(15,16,18 ; 21,24,28),(15,19,24 ; 26,3,9),(16,17,19 ; 22,25,1)$, $(16,20,25 ; 27,4,10),(17,18,20 ; 23,26,2),(17,21,26 ; 28,5,11)$, $(18,19,21 ; 24,27,3),(18,22,27 ; 1,6,12),(19,20,22 ; 25,28,4)$, $(19,23,28 ; 2,7,13),(20,21,23 ; 26,1,5),(21,22,24 ; 27,2,6)$, $(22,23,25 ; 28,3,7),(23,24,26 ; 1,4,8),(24,25,27 ; 2,5,9),(25,26,28 ; 3,6,10)\}$.

Lemma 3.4. $3 S S(n)$ exists if $n=40,52,64$.

Proof. $K_{40}$ can be viewed as the union of two $K_{12}$ 's, one $K_{16}$ and one $K_{12,12,16}$. $K_{52}$ can be viewed as the union of two $K_{12}$ 's, one $K_{28}$ and one $K_{12,12,28} . K_{64}$ can be viewed as the union of one $K_{16}$, two $K_{24}$ 's and one $K_{24,24,16}$. According to Example 3.1 and 3.3, $K_{12}, K_{16}, K_{24}$, and $K_{28}$ can be decomposed into 3-suns. By Lemma $2.5, K_{12,12,16}, K_{12,12,28}$, and $K_{24,24,16}$ can be decomposed into 3 -suns. Hence, $K_{n}$ can be decomposed into 3 -suns for $n=40,52,64$.

Lemma 3.5. If $n \equiv 4(\bmod 12)$, then there exists a 3-sun system of order $n$. 
Proof. Let $n=12 m+4$. By Example 3.3 and Lemma 3.4, we have $3 S S(16)$, $3 S S(28), 3 S S(40), 3 S S(52)$, and $3 S S(64)$. Let $m=3 s+p$ where $s \geq 2$ and $0 \leq p \leq 2 . K_{36 s+12 p+4}$ can be viewed as the union of two $K_{12 s}$ 's, one $K_{12(s+p)+4}$ and one $K_{12 s, 12 s, 12(s+p)+4}$. By Lemma 3.2 and recursive construction, $K_{12 s}$ and $K_{12(s+p)+4}$ can be decomposed into 3 -suns. By Lemma $2.5, K_{12 s, 12 s, 12(s+p)+4}$ can be decomposed into 3 -suns. Hence, the proof is completed.

Next, we will construct cyclic 3 -sun systems of order $n$ for $n \equiv 1(\bmod 12)$. A 3 -sun system $3 S S(n)$ on the elements of $Z_{n}=\{1,2, \ldots, n\}$ is said to be cyclic if whenever $(a, b, c ; x, y, z)$ is a 3 -sun, so also is $(a+1, b+1, c+1 ; x+1, y+1, z+1)$.

\section{Example 3.6.}

(a) The 3 -suns $(i, i+1, i+3 ; i+4, i+6, i+9), 1 \leq i \leq 13$, form a cyclic $3 S S(13)$.

(b) The 3 -suns $(i, i+1, i+5 ; i+9, i+12, i+17),(i, i+2, i+8 ; i+13, i+16, i+23)$, $(i, i+3, i+10 ; i+16, i+20, i+28), 1 \leq i \leq 37$, form a cyclic $3 S S(37)$.

By [1,5], suppose that $\{1,2, \ldots, 3 m\}$ can be partitioned into $m$ triples $\{a, b, c\}$ such that $a+b=c$ or $a+b+c \equiv 0(\bmod 6 m+1)$. Then the triples $\{0, a, a+b\}$ form a $(6 m+1,3,1)$ difference system and so lead to the construction of cyclic $S T S(6 m+1)$. A Skolem triple system of order $m$ is a partition of $\{1,2, \ldots, 3 m\}$ into $m$ triples $\left\{i, a_{i}, i+a_{i}\right\}, 1 \leq i \leq m$. An $O^{\prime}$ Keefe triple system of order $m$ is a partition of $\{1,2, \ldots, 3 m-1,3 m+1\}$ into $m$ triples $\left\{i, a_{i}, i+a_{i}\right\}, 1 \leq i \leq m$. It is well-known that if $m \equiv 0,1(\bmod 4)$ then a Skolem triple system of order $m$ exists and if $m \equiv 2,3(\bmod 4)$ then an O'Keefe triple system of order $m$ exists. Let $t=(a, b, c ; d, e, f)$ be a 3 -sun in $K_{n}$. Since $t$ contains a triangle $(a, b, c)$ and one 1factor $\{\{a, d\},\{b, e\},\{c, f\}\}$, we obtain two difference triples $\{b-a, c-b, a-c\}$ and $\{d-a, e-b, f-c\}$ where the values are taken modulo $n$. Next, we will metamorphose a cyclic $S T S(12 m+1)$ into a cyclic $3 S S(12 m+1)$.

Lemma 3.7. If $n \equiv 1(\bmod 12)$, then there exists a cyclic $3 S S(n)$.

Proof. Let $n=12 k+1$.

(1) $k$ is even.

If $k=2$, then the 3 -suns in a cyclic $3 S S(25)$ are constructed as follows.

For $i=1,2, \ldots, 25$,

$(i, i+1, i+12 ; i+2, i+8, i+21)$ and $(i, i+3, i+8 ; i+4, i+9, i+18)$.

It is easy to check that the union of four difference triples is the set $\{1,2, \ldots, 12\}$.

If $k \geq 4$, then the 3 -suns are constructed as follows.

For $i=1,2, \ldots, n$,

$(i, i+1, i+6 k ; i+k, i+4 k, i+11 k-1)$, 
$\left(i, i+2 k-1, i-1+\frac{9 k}{2} ; i+2 k, i+5 k-1, i-1+\frac{19 k}{2}\right)$, and $j=1,2, \ldots, \frac{k}{2}-1$,

$(i, i+2 j, i+3 k+j ; i+2 j+1, i+5 k+j-1, i+8 k+2 j)$,

$\left(i, i+k+2 j-1, i+\frac{7 k}{2}+j-1 ; i+k+2 j, i+\frac{11 k}{2}+j-2, i+9 k+2 j-2\right)$.

Since from each 3 -sun we can get two difference triples, these difference triples form a Skolem triple system of order $2 k$ when $k$ is even and $k \geq 2$, see[1]. Therefore, we have a cyclic $3 S S(12 k+1), k$ is even.

(2) $k$ is odd.

In Example 3.6, we have a cyclic $3 S S(13)$ and a cyclic $3 S S(37)$. We consider when $k \geq 5$, the 3 -suns are constructed as follows.

For $i=1,2, \ldots, n$,

$(i, i+2 j, i+3 k+j+1 ; i+2 j+1, i+5 k+j-1, i+8 k+2 j+1)$, where $j=1,2, \ldots, \frac{k-1}{2}$.

$\left(i, i+k+2 j-1, i+\frac{7 k+1}{2}+j ; i+k+2 j+2, i+\frac{11 k-3}{2}+j, i+9 k+2 j+2\right)$, where $j=1,2, \ldots, \frac{k-5}{2}$.

$(i, i+2 k-1, i+5 k ; i+2 k-4, i+4 k+2, i+9 k-1)$,

$(i, i+k+2, i+6 k+1 ; i+2 k-2, i+3 k+4, i+10 k+1)$, and

$\left(i, i+1, i+\frac{11 k+3}{2} ; i+2 k, i+2 k+2, i+\frac{19 k+5}{2}\right)$

Since from each 3 -sun we can get two difference triples, these difference triples form an $O^{\prime}$ Keefe triple system of order $2 k$ when $k$ is odd and $k \geq 5$, see[1]. Therefore, we have a cyclic $3 S S(12 k+1), k$ is odd.

Next we will metamorphose a $K T S(12 k+9)$ into a $3 S S(12 k+9)$. A parallel class in a Steiner triple system $(S, T)$ is a set of triples in $T$ that partitions $S$. If the triples in $T$ can be partitioned into parallel classes, then we say $S T S(v)$ is a Kirkman triple system of order $v$, denoted by $K T S(v)$. It is well-known [2] that there exists a $K T S(v)$ if and only if $v \equiv 3(\bmod 6)$.

Lemma 3.8. If $n \equiv 9(\bmod 12)$, then there is a 3 -sun system of order $n$.

Proof. Let $n=12 k+9$ where $k \geq 0$. Then there exists a $K T S(12 k+9)$ with $6 k+4$ parallel classes. Let $(S, T)$ be a $K T S(12 k+9) . \pi$ and $\pi^{\prime}$ are any two distinct parallel classes in $T$. Consider $\pi \cup \pi^{\prime}$. If $(x, y, z) \in \pi$ and $(x, a, b) \in \pi^{\prime}$, then the edges $\{x, y\},\{y, z\}$, and $\{x, z\}$ can not be contained in any triple in $\pi^{\prime}$. That means $y, z, a$, and $b$ are distinct. Using this property, we give a direction to each edge, such that each triple $(x, a, b)$ in $\pi^{\prime}$ forms a directed cycle $\langle x, a, b\rangle$ with the edge set $\{(x, a),(a, b),(b, x)\}$. Similarly, we have $\langle y, c, d\rangle$ and $\langle z, e, f\rangle$ in $\pi^{\prime}$. Any triangle in $\pi$ with its out-edge from $\pi^{\prime}$ forms a 3 -sun. Thus we can get a 3 -sun $(x, y, z ; a, c, e)$ in $\pi \cup \pi^{\prime}$. Therefore, the edge-set of the union of any two distinct parallel classes of $K T S(12 k+9)$ can be decomposed into $4 k+33$-suns. Hence, we obtain a 3 -sun system of order $n$. 
Example 3.9. There is a $3 S S(9)$ constructed from a $K T S(9)$.

Proof. Let $\left(Z_{9}, T\right)$ be a $K T S(9)$ with 4 parallel classes $\pi_{1}, \pi_{2}, \pi_{3}$, and $\pi_{4}$, where $\pi_{1}=\{(1,2,3),(4,5,6),(7,8,9)\}, \pi_{2}=\{(1,4,7),(2,5,8),(3,6,9)\}, \pi_{3}=$ $\{(1,5,9),(2,6,7),(3,4,8)\}$, and $\pi_{4}=\{(1,6,8),(2,4,9),(3,5,7)\}$. We give a direction to each edge in $\pi_{2}$ and $\pi_{4}$ as follows: $\pi_{2}^{\prime}=\{\langle 1,4,7\rangle,\langle 2,5,8\rangle,\langle 3,6,9\rangle\}, \pi_{4}^{\prime}=$ $\{\langle 1,6,8\rangle,\langle 2,4,9\rangle,\langle 3,5,7\rangle\}$. Then the edge-set of $\pi_{1} \cup \pi_{2}^{\prime}$ can be decomposed into three 3 -suns, $(1,2,3 ; 4,5,6),(4,5,6 ; 7,8,9)$, and $(7,8,9 ; 1,2,3) . \pi_{3} \cup \pi_{4}^{\prime}$ can be decomposed into three 3 -suns, $(1,5,9 ; 6,7,2),(2,6,7 ; 4,8,3)$, and $(3,4,8 ; 5,9,1)$.

By Lemma 3.2, 3.5, 3.7, and 3.8, we obtain the following theorem.

Theorem 3.10. There exists a 3-sun system of order $n$, if and only if $n \equiv$ $0,1,4,9(\bmod 12)$.

\section{Embedding A Cyclic Steiner Triple System in a 3-Sun System}

Let $(Y, S)$ be a 3 -sun system of order $n$ and $P$ be the collection of triangles in $S$. Then $(Y, P)$ is a partial triple system of order $n$. We say that the Steiner triple system $(X, T)$ is embedded in a 3 -sun system $(Y, S)$ provided $X \subseteq Y$ and $T \subseteq P$. Subsequently, we give a construction for a 3 -sun system of order $12 m+1$ embedding a cyclic Steiner triple system of order $6 m+1$.

Theorem 4.11. Let $m$ be a positive integer. Let $(X, T)$ be a cyclic Steiner triple system of order $6 m+1$. Then there is a 3-sun system $(Y, S)$ of order $12 m+1$, such that $(X, T)$ is embedded in $(Y, S)$.

Proof. Let $X=\left\{v_{1}, v_{2}, \ldots, v_{6 m}, v_{6 m+1}\right\}, U=\left\{u_{1}, u_{2}, \ldots, u_{6 m}\right\}$ and $X \cap U$ $=\emptyset$. Set $Y=X \cup U$. Let $(X, T)$ be a cyclic $S T S(6 m+1)$. Suppose $E_{1}, E_{2}, \ldots$, and $E_{m}$ are base triples in $T$. For convenience, we give an order for the elements in each base triple such that $E_{i}=\left\langle v_{a_{i}^{1}}, v_{a_{i}^{2}}, v_{a_{i}^{3}}\right\rangle$, for all $i=1,2, \ldots, m$, and $a_{i}^{1}<a_{i}^{2}<a_{i}^{3}$.

Define a collection $S$ of 3 -suns over $Y$ as follows:

(1) For $i=1,2, \ldots, m, j=0,1,2, \ldots, 6 m$.

Define $t_{i, j}^{k}:=a_{i}^{k}+j \in Z_{6 m+1}=\{1,2, \ldots, 6 m+1\}$, for all $k=1,2,3$.

$B_{i, j}:=\left(v_{t_{i, j}^{1}}, v_{t_{i, j}^{2}}, v_{t_{i, j}^{3}} ; u_{2 m+3 i+t_{i, j}^{1}-3}, u_{2 m+3 i+t_{i, j}^{2}-2}, u_{2 m+3 i+t_{i, j}^{3}-1}\right)$ where the indices of $u$ are restricted to $Z_{6 m}=\{1,2, \ldots, 6 m-1,6 m\}$.

Therefore, there are $m(6 m+1) 3$-suns.

(2) Define $\alpha_{k}=v_{k}, k=1,2, \ldots, 6 m$.

For $i=1,2, \ldots, m-1$ and $j=0,1,2, \ldots, 6 m-1$,

$B_{i, j}^{\prime}=\left(u_{1+j}, u_{2 m-2(i-1)+j}, \alpha_{2-i+j} ; u_{2 m+1-2(i-1)+j}, u_{4 m+1-(i-1)+j}, u_{5 m+1+j}\right)$

And $B_{m, j}^{\prime}:=\left(u_{1+j}, u_{2+j}, \alpha_{5 m+2+j} ; u_{3+j}, \beta_{j}, u_{5 m+1+j}\right)$ where

$$
\beta_{j}:= \begin{cases}v_{6 m+1} & \text { if } j=0,1,2, \ldots, 2 m-2,5 m-1,5 m, \ldots, 6 m-1 . \\ u_{3 m+2+j} & \text { if } j=2 m-1,2 m, \ldots, 5 m-3,5 m-2 .\end{cases}
$$


The indices of $\alpha$ and $u$ are restricted to $Z_{6 m}=\{1,2, \ldots, 6 m\}$. Hence, there are $6 m^{2} 3$-suns.

From (1), the base triples in $T$ is the triangles of $B_{i, 0}$, for $i=1,2, \ldots, m$. Therefore, $(X, T)$ is embedded in $(Y, S)$.

Example 4.12. Let $X=\left\{v_{1}, v_{2}, \ldots, v_{7}\right\}, U=\left\{u_{1}, u_{2}, \ldots, u_{6}\right\}$ and $Y=X \cup$ $U$. Let $(X, T)$ be a cyclic $S T S(7)$. If $\left\{v_{1}, v_{2}, v_{4}\right\}$ is a base triple in $T$. Let $E_{i}=\left\langle v_{a_{1}^{1}}, v_{a_{1}^{2}}, v_{a_{1}^{3}}\right\rangle=\left\langle v_{1}, v_{2}, v_{4}\right\rangle$, and $S=\left\{B_{1, j}, B_{1, j^{\prime}}^{\prime} \mid j=0,1, \ldots, 6, j^{\prime}=\right.$ $0,1, \ldots, 5\}$. By the construction in Theorem 4.1, we can get:

$B_{1,0}=\left(v_{1}, v_{2}, v_{4} ; u_{3}, u_{5}, u_{2}\right), B_{1,1}=\left(v_{2}, v_{3}, v_{5} ; u_{4}, u_{6}, u_{2}\right), B_{1,2}=\left(v_{3}, v_{4}, v_{6} ; u_{5}, u_{1}, u_{4}\right)$, $B_{1,3}=\left(v_{4}, v_{5}, v_{7} ; u_{6}, u_{2}, u_{5}\right), B_{1,4}=\left(v_{5}, v_{6}, v_{1} ; u_{1}, u_{3}, u_{5}\right), B_{1,5}=\left(v_{6}, v_{7}, v_{2} ; u_{2}, u_{4}, u_{6}\right)$, $B_{1,6}=\left(v_{7}, v_{1}, v_{3} ; u_{3}, u_{4}, u_{1}\right), B_{1,0}^{\prime}=\left(u_{1}, u_{2}, v_{1} ; u_{3}, v_{7}, u_{6}\right), B_{1,1}^{\prime}=\left(u_{2}, u_{3}, v_{2} ; u_{4}, u_{6}, u_{1}\right)$, $B_{1,2}^{\prime}=\left(u_{3}, u_{4}, v_{3} ; u_{5}, u_{1}, u_{2}\right), B_{1,3}^{\prime}=\left(u_{4}, u_{5}, v_{4} ; u_{6}, u_{2}, u_{3}\right), B_{1,4}^{\prime}=\left(u_{5}, u_{6}, v_{5} ; u_{1}, v_{7}, u_{4}\right)$, $B_{1,5}^{\prime}=\left(u_{6}, u_{1}, v_{6} ; u_{2}, v_{7}, u_{5}\right)$. Then $(Y, S)$ is a $3 S S(13)$ and $(X, T)$ is a cyclic $\operatorname{STS}(7)$ embedded in a $3 S S(13)$.

\section{Conclusion And Open Question}

There are further questions to be asked.

(1) If $p>q>r \geq 2$, what is the necessary and sufficient condition for the decomposition of $K_{p, q, r}$ into 3-suns ?

(2) Can one embed any Steiner triple system into a 3-sun system?

\section{APPENDIX}

A. $K_{6,6,3}$ can be decomposed into 123 -suns as follows:

$\left\{\left(a_{1}, b_{1}, c_{1} ; b_{2}, a_{2}, b_{3}\right),\left(a_{2}, b_{2}, c_{2} ; b_{3}, a_{3}, b_{1}\right),\left(a_{3}, b_{3}, c_{3} ; b_{1}, a_{1}, b_{2}\right)\right.$, $\left(a_{4}, b_{4}, c_{1} ; b_{5}, a_{5}, a_{2}\right),\left(a_{5}, b_{5}, c_{2} ; b_{6}, a_{6}, a_{3}\right),\left(a_{6}, b_{6}, c_{3} ; b_{4}, a_{4}, a_{1}\right)$, $\left(a_{4}, b_{1}, c_{3} ; b_{2}, a_{5}, b_{4}\right),\left(a_{5}, b_{2}, c_{1} ; b_{3}, a_{6}, b_{5}\right),\left(a_{6}, b_{3}, c_{2} ; b_{1}, a_{4}, b_{6}\right)$, $\left.\left(a_{1}, b_{4}, c_{2} ; b_{5}, a_{2}, a_{4}\right),\left(a_{2}, b_{5}, c_{3} ; b_{6}, a_{3}, a_{5}\right),\left(a_{3}, b_{6}, c_{1} ; b_{4}, a_{1}, a_{6}\right)\right\}$.

B. $K_{6,6,4}$ can be decomposed into 143 -suns as follows:

$\left\{\left(a_{1}, b_{1}, c_{1} ; b_{2}, c_{4}, a_{3}\right),\left(a_{2}, b_{2}, c_{2} ; b_{3}, c_{3}, a_{3}\right),\left(a_{3}, b_{3}, c_{3} ; b_{1}, a_{4}, b_{4}\right)\right.$, $\left(a_{4}, b_{4}, c_{1} ; c_{4}, a_{6}, b_{5}\right),\left(a_{6}, b_{5}, c_{2} ; c_{4}, a_{5}, b_{6}\right),\left(a_{6}, b_{6}, c_{3} ; b_{2}, a_{5}, b_{1}\right)$, $\left(a_{4}, b_{1}, c_{2} ; c_{3}, a_{5}, b_{3}\right),\left(a_{5}, b_{2}, c_{4} ; b_{4}, a_{4}, b_{3}\right),\left(a_{6}, b_{3}, c_{1} ; b_{1}, a_{5}, b_{2}\right)$, $\left(a_{1}, b_{4}, c_{2} ; b_{3}, a_{3}, a_{5}\right),\left(a_{2}, b_{5}, c_{3} ; b_{1}, a_{3}, a_{5}\right),\left(a_{3}, b_{6}, c_{4} ; b_{2}, a_{1}, a_{2}\right)$, $\left.\left(a_{1}, b_{5}, c_{4} ; c_{3}, a_{4}, b_{4}\right),\left(a_{2}, b_{6}, c_{1} ; b_{4}, a_{4}, a_{5}\right)\right\}$.

C. $K_{6,6,5}$ can be decomposed into 163 -suns as follows:

$\left\{\left(a_{1}, b_{1}, c_{1} ; b_{2}, a_{2}, b_{5}\right),\left(a_{2}, b_{2}, c_{2} ; b_{3}, a_{3}, b_{6}\right),\left(a_{3}, b_{3}, c_{3} ; c_{2}, a_{4}, a_{1}\right)\right.$, $\left(a_{4}, b_{4}, c_{4} ; b_{5}, a_{1}, a_{5}\right),\left(a_{2}, b_{5}, c_{4} ; b_{6}, a_{6}, b_{1}\right),\left(a_{3}, b_{6}, c_{4} ; c_{1}, a_{5}, b_{2}\right)$, $\left(a_{4}, b_{1}, c_{2} ; c_{3}, a_{3}, b_{5}\right),\left(a_{6}, b_{1}, c_{3} ; c_{2}, a_{5}, b_{6}\right),\left(a_{4}, b_{2}, c_{5} ; c_{1}, c_{3}, a_{2}\right)$, 
$\left(a_{6}, b_{2}, c_{1} ; c_{4}, a_{5}, b_{4}\right),\left(a_{5}, b_{3}, c_{1} ; c_{2}, a_{6}, b_{6}\right),\left(a_{5}, b_{5}, c_{5} ; c_{3}, a_{3}, b_{3}\right)$, $\left(a_{6}, b_{4}, c_{5} ; b_{6}, a_{5}, b_{1}\right),\left(a_{2}, b_{4}, c_{3} ; c_{1}, a_{3}, b_{5}\right),\left(a_{1}, b_{3}, c_{2} ; b_{5}, c_{4}, b_{4}\right)$, $\left.\left(a_{1}, b_{6}, c_{5} ; c_{4}, a_{4}, a_{3}\right)\right\}$.

D. $K_{6,6,7}$ can be decomposed into 203 -suns as follows: $\left\{\left(a_{1}, b_{1}, c_{1} ; c_{7}, a_{4}, a_{5}\right),\left(a_{2}, b_{2}, c_{2} ; c_{1}, a_{1}, a_{4}\right),\left(a_{3}, b_{3}, c_{3} ; b_{4}, a_{2}, a_{5}\right)\right.$, $\left(a_{4}, b_{4}, c_{4} ; b_{6}, a_{1}, b_{5}\right),\left(a_{5}, b_{5}, c_{5} ; b_{2}, a_{2}, a_{1}\right),\left(a_{6}, b_{6}, c_{6} ; c_{3}, c_{1}, a_{2}\right)$, $\left(a_{2}, b_{1}, c_{3} ; c_{5}, a_{5}, b_{6}\right),\left(a_{3}, b_{2}, c_{4} ; b_{6}, c_{7}, b_{1}\right),\left(a_{4}, b_{3}, c_{5} ; c_{3}, c_{6}, b_{4}\right)$, $\left(a_{5}, b_{4}, c_{6} ; b_{6}, a_{2}, a_{1}\right),\left(a_{6}, b_{5}, c_{7} ; c_{5}, a_{4}, b_{4}\right),\left(a_{3}, b_{1}, c_{5} ; c_{2}, c_{6}, b_{2}\right)$, $\left(a_{4}, b_{2}, c_{6} ; c_{7}, c_{3}, a_{3}\right),\left(a_{5}, b_{3}, c_{7} ; c_{2}, c_{1}, b_{6}\right),\left(a_{6}, b_{4}, c_{1} ; b_{2}, c_{3}, a_{4}\right)$, $\left(a_{1}, b_{5}, c_{2} ; b_{6}, c_{3}, b_{4}\right),\left(a_{2}, b_{6}, c_{4} ; c_{7}, c_{5}, a_{6}\right),\left(a_{6}, b_{1}, c_{2} ; b_{3}, c_{7}, b_{6}\right)$, $\left.\left(a_{1}, b_{3}, c_{4} ; c_{3}, c_{2}, a_{5}\right),\left(a_{3}, b_{5}, c_{1} ; c_{7}, c_{6}, b_{2}\right)\right\}$.

E. $K_{6,6,8}$ can be decomposed into 223 -suns as follows: $\left\{\left(a_{1}, b_{1}, c_{1} ; b_{2}, c_{8}, a_{2}\right),\left(a_{2}, b_{2}, c_{2} ; b_{4}, c_{1}, a_{6}\right),\left(a_{3}, b_{3}, c_{3} ; c_{6}, a_{2}, a_{6}\right)\right.$, $\left(a_{4}, b_{4}, c_{4} ; b_{1}, c_{1}, a_{5}\right),\left(a_{5}, b_{5}, c_{5} ; c_{8}, a_{2}, b_{6}\right),\left(a_{6}, b_{6}, c_{6} ; b_{2}, c_{3}, a_{2}\right)$, $\left(a_{2}, b_{1}, c_{3} ; c_{5}, c_{7}, a_{1}\right),\left(a_{3}, b_{2}, c_{4} ; c_{7}, c_{5}, b_{1}\right),\left(a_{4}, b_{3}, c_{5} ; b_{5}, c_{1}, b_{4}\right)$, $\left(a_{5}, b_{4}, c_{6} ; c_{1}, c_{2}, a_{1}\right),\left(a_{6}, b_{5}, c_{7} ; b_{3}, c_{3}, b_{4}\right),\left(a_{1}, b_{6}, c_{8} ; c_{5}, a_{3}, a_{2}\right)$, $\left(a_{3}, b_{1}, c_{5} ; c_{8}, c_{2}, a_{6}\right),\left(a_{4}, b_{2}, c_{6} ; c_{1}, c_{8}, b_{1}\right),\left(a_{5}, b_{3}, c_{7} ; b_{6}, c_{6}, a_{4}\right)$, $\left(a_{6}, b_{4}, c_{8} ; b_{1}, a_{3}, a_{4}\right),\left(a_{1}, b_{5}, c_{2} ; c_{7}, c_{8}, a_{5}\right),\left(a_{2}, b_{6}, c_{4} ; c_{7}, c_{1}, a_{6}\right)$, $\left(a_{3}, b_{5}, c_{1} ; c_{2}, c_{6}, a_{6}\right),\left(a_{4}, b_{6}, c_{2} ; c_{3}, c_{7}, b_{3}\right),\left(a_{1}, b_{3}, c_{4} ; b_{4}, c_{8}, b_{5}\right)$, $\left.\left(a_{5}, b_{2}, c_{3} ; b_{1}, c_{7}, b_{4}\right)\right\}$.

F. $K_{6,6,10}$ can be decomposed into 263 -suns as follows:

$\left\{\left(a_{1}, b_{1}, c_{1} ; b_{3}, c_{8}, b_{6}\right),\left(a_{2}, b_{2}, c_{2} ; b_{4}, a_{1}, a_{4}\right),\left(a_{3}, b_{3}, c_{3} ; c_{1}, c_{2}, b_{4}\right)\right.$, $\left(a_{4}, b_{4}, c_{4} ; c_{3}, c_{1}, b_{5}\right),\left(a_{5}, b_{5}, c_{5} ; c_{2}, c_{3}, b_{6}\right),\left(a_{6}, b_{6}, c_{6} ; c_{4}, c_{3}, b_{5}\right)$, $\left(a_{2}, b_{1}, c_{3} ; b_{3}, a_{6}, a_{1}\right),\left(a_{3}, b_{2}, c_{4} ; b_{5}, c_{9}, a_{2}\right),\left(a_{4}, b_{3}, c_{5} ; c_{1}, c_{4}, b_{4}\right)$, $\left(a_{5}, b_{4}, c_{6} ; c_{4}, c_{2}, b_{3}\right),\left(a_{6}, b_{5}, c_{7} ; c_{3}, c_{8}, a_{3}\right),\left(a_{1}, b_{6}, c_{8} ; c_{4}, c_{7}, a_{4}\right)$, $\left(a_{3}, b_{1}, c_{5} ; b_{4}, c_{2}, b_{2}\right),\left(a_{4}, b_{2}, c_{6} ; b_{6}, c_{1}, b_{1}\right),\left(a_{5}, b_{3}, c_{7} ; c_{1}, c_{8}, a_{2}\right)$, $\left(a_{6}, b_{4}, c_{8} ; c_{2}, c_{7}, a_{3}\right),\left(a_{1}, b_{5}, c_{9} ; c_{5}, c_{10}, a_{2}\right),\left(a_{2}, b_{6}, c_{10} ; c_{5}, c_{9}, a_{5}\right)$, $\left(a_{4}, b_{1}, c_{7} ; b_{5}, c_{4}, a_{1}\right),\left(a_{5}, b_{2}, c_{8} ; b_{6}, c_{3}, a_{2}\right),\left(a_{6}, b_{3}, c_{9} ; c_{1}, c_{10}, a_{4}\right)$, $\left(a_{1}, b_{4}, c_{10} ; c_{6}, c_{9}, a_{4}\right),\left(a_{2}, b_{5}, c_{1} ; c_{6}, c_{2}, b_{3}\right),\left(a_{3}, b_{6}, c_{2} ; c_{6}, c_{4}, a_{1}\right)$, $\left.\left(a_{5}, b_{1}, c_{9} ; c_{3}, c_{10}, a_{3}\right),\left(a_{6}, b_{2}, c_{10} ; c_{5}, c_{7}, a_{3}\right)\right\}$.

G. $K_{6,6,11}$ can be decomposed into 283 -suns as follows:

$\left\{\left(a_{1}, b_{1}, c_{1} ; b_{2}, c_{8}, b_{5}\right),\left(a_{2}, b_{2}, c_{2} ; c_{7}, c_{11}, a_{4}\right),\left(a_{3}, b_{3}, c_{3} ; c_{11}, c_{2}, b_{4}\right)\right.$, $\left(a_{4}, b_{4}, c_{4} ; c_{3}, c_{11}, b_{5}\right),\left(a_{5}, b_{5}, c_{5} ; c_{11}, c_{3}, b_{6}\right),\left(a_{6}, b_{6}, c_{6} ; c_{3}, c_{4}, b_{5}\right)$, $\left(a_{2}, b_{1}, c_{3} ; b_{3}, a_{6}, a_{1}\right),\left(a_{3}, b_{2}, c_{4} ; b_{5}, c_{9}, a_{2}\right),\left(a_{4}, b_{3}, c_{5} ; c_{11}, c_{4}, b_{4}\right)$, $\left(a_{5}, b_{4}, c_{6} ; c_{4}, c_{2}, b_{3}\right),\left(a_{6}, b_{5}, c_{7} ; c_{4}, c_{8}, b_{6}\right),\left(a_{1}, b_{6}, c_{8} ; c_{4}, c_{11}, a_{4}\right)$, $\left(a_{3}, b_{1}, c_{5} ; c_{1}, c_{2}, b_{2}\right),\left(a_{4}, b_{2}, c_{6} ; b_{6}, c_{1}, b_{1}\right),\left(a_{5}, b_{3}, c_{7} ; c_{1}, c_{8}, a_{3}\right)$, $\left(a_{6}, b_{4}, c_{8} ; c_{2}, c_{7}, a_{3}\right),\left(a_{1}, b_{5}, c_{9} ; c_{5}, c_{10}, b_{6}\right),\left(a_{2}, b_{6}, c_{10} ; c_{5}, c_{3}, a_{5}\right)$, $\left(a_{4}, b_{1}, c_{7} ; b_{5}, c_{4}, a_{1}\right),\left(a_{5}, b_{2}, c_{8} ; b_{6}, c_{3}, a_{2}\right),\left(a_{6}, b_{3}, c_{9} ; c_{1}, c_{10}, a_{4}\right)$, $\left(a_{1}, b_{4}, c_{10} ; c_{6}, c_{9}, a_{4}\right),\left(a_{2}, b_{5}, c_{11} ; c_{6}, c_{2}, a_{6}\right),\left(a_{3}, b_{6}, c_{2} ; c_{6}, c_{1}, a_{5}\right)$, $\left(a_{5}, b_{1}, c_{9} ; c_{3}, c_{10}, a_{3}\right),\left(a_{6}, b_{2}, c_{10} ; c_{5}, c_{7}, a_{3}\right),\left(a_{1}, b_{3}, c_{11} ; c_{2}, c_{1}, b_{1}\right)$, 
$\left.\left(a_{2}, b_{4}, c_{1} ; c_{9}, a_{3}, a_{4}\right)\right\}$.

H. $K_{6,6,13}$ can be decomposed into 323 -suns as follows:

$\left\{\left(a_{1}, b_{1}, c_{1} ; c_{13}, c_{2}, a_{4}\right),\left(a_{2}, b_{2}, c_{2} ; c_{1}, c_{3}, b_{3}\right),\left(a_{3}, b_{3}, c_{3} ; b_{4}, c_{4}, a_{5}\right)\right.$, $\left(a_{4}, b_{4}, c_{4} ; c_{8}, c_{1}, b_{1}\right),\left(a_{5}, b_{5}, c_{5} ; c_{10}, c_{2}, a_{6}\right),\left(a_{6}, b_{6}, c_{6} ; c_{12}, a_{5}, a_{3}\right)$, $\left(a_{2}, b_{1}, c_{3} ; b_{3}, c_{12}, a_{4}\right),\left(a_{3}, b_{2}, c_{4} ; c_{8}, c_{1}, a_{5}\right),\left(a_{4}, b_{3}, c_{5} ; c_{9}, c_{6}, b_{6}\right)$, $\left(a_{5}, b_{4}, c_{6} ; c_{13}, c_{2}, a_{2}\right),\left(a_{6}, b_{5}, c_{7} ; c_{13}, c_{3}, a_{3}\right),\left(a_{1}, b_{6}, c_{8} ; c_{3}, c_{1}, a_{2}\right)$, $\left(a_{3}, b_{1}, c_{5} ; c_{1}, c_{6}, b_{2}\right),\left(a_{4}, b_{2}, c_{6} ; c_{11}, c_{7}, a_{1}\right),\left(a_{5}, b_{3}, c_{7} ; c_{12}, c_{1}, a_{2}\right)$, $\left(a_{6}, b_{4}, c_{8} ; c_{1}, c_{3}, b_{5}\right),\left(a_{1}, b_{5}, c_{9} ; c_{2}, c_{4}, a_{2}\right),\left(a_{2}, b_{6}, c_{10} ; c_{4}, c_{3}, a_{4}\right)$, $\left(a_{4}, b_{1}, c_{7} ; c_{13}, c_{8}, b_{6}\right),\left(a_{5}, b_{2}, c_{8} ; c_{1}, c_{9}, b_{3}\right),\left(a_{6}, b_{3}, c_{9} ; c_{2}, c_{10}, b_{4}\right)$, $\left(a_{1}, b_{4}, c_{10} ; c_{4}, c_{5}, a_{3}\right),\left(a_{2}, b_{5}, c_{11} ; c_{13}, c_{6}, b_{4}\right),\left(a_{3}, b_{6}, c_{12} ; c_{9}, c_{4}, a_{4}\right)$, $\left(a_{5}, b_{1}, c_{9} ; c_{2}, c_{10}, b_{6}\right),\left(a_{6}, b_{2}, c_{10} ; c_{3}, c_{11}, b_{5}\right),\left(a_{1}, b_{3}, c_{11} ; c_{5}, c_{13}, b_{6}\right)$, $\left(a_{2}, b_{4}, c_{12} ; c_{5}, c_{7}, b_{3}\right),\left(a_{3}, b_{5}, c_{13} ; c_{11}, c_{1}, b_{4}\right),\left(a_{4}, b_{6}, c_{2} ; b_{5}, c_{13}, a_{3}\right)$, $\left.\left(a_{6}, b_{1}, c_{11} ; c_{4}, c_{13}, a_{5}\right),\left(a_{1}, b_{2}, c_{12} ; c_{7}, c_{13}, b_{5}\right)\right\}$.

I. $K_{6,6,14}$ can be decomposed into 343 -suns as follows: $\left\{\left(a_{1}, b_{1}, c_{1} ; c_{13}, c_{2}, a_{4}\right),\left(a_{2}, b_{2}, c_{2} ; c_{1}, c_{3}, b_{3}\right),\left(a_{3}, b_{3}, c_{3} ; c_{10}, c_{4}, a_{5}\right)\right.$, $\left(a_{4}, b_{4}, c_{4} ; b_{5}, c_{1}, b_{1}\right),\left(a_{5}, b_{5}, c_{5} ; c_{14}, c_{2}, a_{6}\right),\left(a_{6}, b_{6}, c_{6} ; c_{12}, a_{5}, a_{3}\right)$, $\left(a_{2}, b_{1}, c_{3} ; c_{9}, c_{12}, a_{4}\right),\left(a_{3}, b_{2}, c_{4} ; c_{2}, c_{1}, a_{5}\right),\left(a_{4}, b_{3}, c_{5} ; c_{9}, c_{6}, b_{6}\right)$, $\left(a_{5}, b_{4}, c_{6} ; c_{13}, c_{2}, a_{2}\right),\left(a_{6}, b_{5}, c_{7} ; c_{13}, c_{3}, a_{3}\right),\left(a_{1}, b_{6}, c_{8} ; c_{3}, c_{2}, a_{2}\right)$, $\left(a_{3}, b_{1}, c_{5} ; c_{8}, c_{6}, b_{2}\right),\left(a_{4}, b_{2}, c_{6} ; c_{11}, c_{7}, a_{1}\right),\left(a_{5}, b_{3}, c_{7} ; c_{12}, c_{1}, a_{1}\right)$, $\left(a_{6}, b_{4}, c_{8} ; c_{1}, c_{3}, b_{5}\right),\left(a_{1}, b_{5}, c_{9} ; c_{2}, c_{4}, b_{2}\right),\left(a_{2}, b_{6}, c_{10} ; c_{4}, c_{3}, a_{4}\right)$, $\left(a_{4}, b_{1}, c_{7} ; c_{2}, c_{14}, b_{6}\right),\left(a_{5}, b_{2}, c_{8} ; c_{1}, c_{14}, b_{1}\right),\left(a_{6}, b_{3}, c_{9} ; c_{2}, c_{10}, b_{4}\right)$, $\left(a_{1}, b_{4}, c_{10} ; c_{4}, c_{5}, a_{5}\right),\left(a_{2}, b_{5}, c_{11} ; c_{14}, c_{6}, b_{4}\right),\left(a_{3}, b_{6}, c_{12} ; c_{9}, c_{4}, a_{4}\right)$, $\left(a_{5}, b_{1}, c_{9} ; c_{2}, c_{10}, b_{6}\right),\left(a_{6}, b_{2}, c_{10} ; c_{3}, c_{11}, b_{5}\right),\left(a_{1}, b_{3}, c_{11} ; c_{5}, c_{8}, b_{6}\right)$, $\left(a_{2}, b_{4}, c_{12} ; c_{5}, c_{7}, b_{3}\right),\left(a_{3}, b_{5}, c_{13} ; c_{11}, c_{1}, b_{6}\right),\left(a_{4}, b_{6}, c_{14} ; c_{8}, c_{1}, b_{5}\right)$, $\left(a_{6}, b_{1}, c_{11} ; c_{4}, c_{13}, a_{5}\right),\left(a_{1}, b_{2}, c_{12} ; c_{14}, c_{13}, b_{5}\right),\left(a_{2}, b_{3}, c_{13} ; c_{7}, c_{14}, a_{4}\right)$, $\left.\left(a_{3}, b_{4}, c_{14} ; c_{1}, c_{13}, a_{6}\right)\right\}$.

\section{ACKNOWLEDGMENTS}

The authors would like to thank the referees for their helpful comments.

\section{REFERENCES}

1. I. Anderson, Combinatorial Designs: Construction Methods, Ellis Horwood Limited, 1990.

2. C. J. Colbourn and A. Rosa, Triple Systems, Charendom Press, Oxford, 1999.

3. T. P. Kirkman, On a problem in combinatorics, Cambridge and Dublin Math. J., 2 (1987), 191-204.

4. C. C. Lindner and C. A. Rodger, Design Theorey, CRC Press, 1997.

5. W. D. Wallis, Combinatorial Design, Marcel Dekker, New York and Besel, 1988. 
6. J. X. Yin and B. S. Gong, Existence of G-designs with $|V(G)|=6$, Combinatorial designs and applications (Huangshan, 1988, pp. 201-218), Lecture Notes in Pure and Appl. Math., Vol. 126, Dekker, New York, 1990.

Chin-Mei Fu, Nan-Hua Jhuang, Yuan-Lung Lin and Hsiao-Ming Sung Department of Mathematics

Tamkang University

Tamsui 251, Taipei, Taiwan

E-mail: cmfu@mail.tku.edu.tw

gaussla@yahoo.com.tw 